\title{
Prevenção da Mucosite Oral em Pacientes submetidos à Quimioterapia
}

doi: https://doi.org/10.32635/2176-9745.RBC.2018v64n2.77

\author{
Prevention of Oral Mucositis in Patients undergoing Chemotherapy \\ Prevención de la Mucositis Oral en Pacientes sometidos a la Quimioterapia
}

\section{Francielli Valduga'; Elenusa Oltramari²; Letícia Tainá de Oliveira Lemes ${ }^{3}$; Carlos Eduardo de Mattos ${ }^{4}$; Letícia Stefenon ${ }^{5}$; Carolina Barreto Mozzini ${ }^{6}$}

Resumo

Introduçáo: A quimioterapia é uma das formas de tratar o câncer, na qual utilizam-se drogas como o 5-fluorouracil (5-FU), maior causador da mucosite oral. Essa complicação é a causa mais comum de dor. Objetivo. Avaliar o grau de mucosite oral durante os dez dias após cada ciclo de quimioterapia, segundo a autopercepção de cada paciente e o nível de dor relatada com o uso das duas substâncias estudo: chá de malva e clorexidina $0,12 \%$. Método. Os pacientes foram randomizados por sorteio para a realizaçáo de bochechos com $10 \mathrm{ml}$ da substância determinada, três vezes ao dia, durante o período de infusão da quimioterapia. Em cada ciclo, utilizou-se uma das substâncias. Durante os dez dias após a quimioterapia, os pacientes responderam a um questionário com perguntas fechadas sobre a sua dor e a autopercepção da sua mucosa oral. Resultados. Nos ciclos utilizando o chá de malva, a autopercepçáo do paciente pareceu ser melhor, com sua mucosite oral sendo de graus 1 e 2 . Contudo, nos ciclos utilizando clorexidina $0,12 \%$, os pacientes apresentaram menos dor. Conclusáo. Tanto nos ciclos em que foi utilizado a clorexidina $0,12 \%$ quanto naqueles onde foi utilizado o chá de malva, a maioria dos pacientes referiu apresentar mucosite oral. Porém, quando utilizado o chá de malva, a frequência de mucosite foi em menores graus. A clorexidina $0,12 \%$ pareceu apresentar menos sintomatologia dolorosa, apesar da diferença, comparando as duas substâncias, ter sido pequena.

Palavras-chave: Mucosite; Prevenção \& Controle; Clorexidina; Malva.

\begin{abstract}
Introduction: The chemotherapy is one of the cancer possible treatments and use chemotherapeutic drugs as 5 -fluorouracil (5-FU), major cause of oral mucositis. This complication is the most common cause of pain. There is still no specific protocol for the prevention of this complication, but there are substances used empirically and palliative. Objective: Assessing the degree of mucositis during the 10 days after each chemotherapy cycle using the self-perception of each patient and the pain level reported with the use of the two substances studied: mallow tea and $0,12 \%$ chlorhexidine. Method: The selected patients were randomly randomized to perform mouthwash with $10 \mathrm{ml}$ of the test substance, 3 times a day, during the infusion time of chemotherapy. In each accompanied cycle one of the studied substances were used. During the 10 days after chemotherapy, patients answered a questionnaire with closed questions about their pain and self-perception of their oral mucosa. Results: in cycles where mallow tea was used, selfperception of patients seems to be better with your oral mucositis is grade 1 and 2 . However, in cycles where $0,12 \%$ chlorhexidine was used, patients experienced less pain. Conclusion: In both cycles that was used at $0.12 \%$ chlorhexidine was used as those where the mauve tea, most of the patients reported oral mucositis present. However, when used mauve tea was obtained mucositis in minor degrees. The $0.12 \%$ chlorhexidine it appeared to have less pain symptoms, although the difference was small when compared to the two substances.

Key words: Mucositis; Prevention \& control; Chlorhexidine; Malva.
\end{abstract}

\section{Resumen}

Introducción: La quimioterapia es una forma de tratamiento de cáncer y se utilizan drogas como el 5-fluorouracilo (5-FU), mayor causante de la mucositis oral. Aún no hay un protocolo específico para la prevención de esta complicación. Objetivo: Evaluar el grado de mucositis oral durante los 10 días después de cada ciclo de quimioterapia según la auto-percepción de cada paciente y el nivel de dolor relatado con el uso de las dos sustancias estudio: té de malva y clorexidina $0,12 \%$. Método: Los pacientes fueron aleatorizados por sorteo para la realización de enjuague con $10 \mathrm{ml}$ de la sustancia determinada, 3 veces al día, durante el período de infusión de la quimioterapia. En cada ciclo se utilizó una de las sustancias. Durante los 10 días después de la quimioterapia los pacientes respondieron a un cuestionario con preguntas cerradas sobre su dolor y la autopercepción de su mucosa oral. Resultados: En los ciclos utilizando el té de malva la auto-percepción del paciente pareció ser mejor, con su mucositis oral siendo de grado 1 y 2. En los ciclos utilizando clorexidina $0,12 \%$ los pacientes presentaron menos dolor. Conclusión: Tanto en los ciclos en que se utilizó la clorexidina $0,12 \%$ como en aquellos donde se utilizó el té de malva, la mayoría de los pacientes refirió presentar mucositis oral. Sin embargo, cuando se utilizó el té de malva la frecuencia de mucositis fue en menores grados. La clorexidina $0,12 \%$ pareció presentar menos sintomatología dolorosa, a pesar de la diferencia comparando las dos sustancias haber sido pequeña.

Palabras clave: Mucositis; Prevención \& control; Clorhexidina; Malva.

\footnotetext{
${ }^{1}$ Cirurgiã-Dentista. Especialista em Endodontia. Prefeitura Municipal de Marau. Marau (RS), Brasil. E-mail:franvalduga@hotmail.com. Orcid ID: https://orcid. org/0000-0002-2020-0160

${ }^{2}$ Cirurgiã-Dentista. Programa de Pós-Graduação em Odontologia da Faculdade Meridional (IMED). Passo Fundo (RS), Brasil. E-mail: nuza1992@gmail.com. Orcid ID: https://orcid.org/0000-0001-7509-0803

${ }^{3}$ Cirurgiã-Dentista. Programa de Pós-Graduação em Odontologia da IMED. Passo Fundo (RS), Brasil. E-mail: leticiatlemes@hotmail.com. Orcid ID: https://orcid. org/0000-0002-6525-5368

${ }^{4}$ Técnico de Enfermagem do Serviço de Oncologia do Hospital da Cidade de Passo Fundo (RS), Brasil. E-mail: onco@hcpf.com.br. Orcid ID: https://orcid.org/00000002-1850-9943

${ }^{5}$ Cirurgiã-Dentista. Mestre em Clínica Odontológica. Doutoranda em Clínicas Odontológicas. Docente da Faculdade Especializada na Área de Saúde do Rio Grande do Sul. Passo Fundo (RS), Brasil. E-mail: leticiastefenon@yahoo.com.br. Orcid ID: https://orcid.org/0000-0002-6135-959X

${ }^{6}$ Fisioterapeuta. Doutora em Ciências e Oncologia. Preceptora da Residência Multiprofissional em Saúde do Hospital da Cidade de Passo Fundo. Passo Fundo (RS), Brasil. E-mail: carolmozzini@gmail.com. Orcid ID: https://orcid.org/0000-0003-4360-2936
}

Endereço correspondência: Carolina Barreto Mozzini. Polus Care Centro Clínico. Rua Bento Gonçalves, 900 - Sala 101 - Centro. Passo Fundo (RS), Brasil. CEP 99020-060. 


\section{INTRODUÇÃO}

O câncer é uma das doenças que mais acomete pessoas no mundo inteiro. Uma das modalidades para o seu tratamento é a quimioterapia, a qual utiliza agentes toxicológicos como o 5-fluorouracil (5-FU), sendo uma das drogas que provocam mais danos às células de alta proliferação como as da mucosa oral ${ }^{1,2}$.

A dor apresentada pelo paciente bem como a gravidade da mucosite oral podem comprometer diretamente as dosagens e o esquema terapêutico da quimioterapia, impactando no prognóstico da doença e na sobrevida do paciente ${ }^{2,3,4}$.

O uso da clorexidina $0,12 \%$ é uma alternativa em muitas pesquisas para prevençáo e tratamento da mucosite oral, em função de suas propriedades antifúngicas e antimicrobianas. Essas propriedades também são apontadas para substâncias fitoterápicas como o chá de malva ${ }^{5,6,7,8,9}$. Uma vez instalada, o tratamento padrão para mucosite oral é laserterapia de baixa potência ${ }^{10}$.

Dessa forma, o objetivo desta pesquisa foi avaliar o grau de mucosite oral durante os dez dias após cada ciclo de quimioterapia, segundo a autopercepção do paciente e o nível de dor relatada com o uso das duas substâncias em estudo.

\section{MÉTODO}

Este estudo trata de um ensaio clínico randomizado, no qual os pacientes náo tinham o conhecimento da substância que utilizaram em cada ciclo.

Inicialmente esta pesquisa foi aprovada pelo Hospital da Cidade de Passo Fundo - RS, local de aplicação, e pelo Comitê de Ética em Pesquisa por meio do parecer número 236.127.

Os pacientes foram selecionados pelos pesquisadores, no Serviço de Oncologia do Hospital da Cidade de Passo Fundo - RS, no período de outubro de 2013 a abril de 2014.

Os critérios de inclusão foram: pacientes maiores de 18 anos e de ambos os sexos, portadores de neoplasias, submetidos à quimioterapia de infusão por quatro dias com 5-FU e que concordaram com a participação assinando o Termo de Consentimento Livre e Esclarecido. Foram excluídos os sujeitos submetidos à radioterapia concomitantemente à quimioterapia, fumantes ou etilistas durante o tratamento.

Os pacientes foram randomizados por sorteio para determinar a primeira substância a ser utilizada na profilaxia, sendo que, em cada ciclo, receberam uma das substâncias: clorexidina $0,12 \%$ ou chá de malva, alternadamente, para realização da profilaxia da mucosite oral, durante os quatro dias de infusão da quimioterapia. O sorteio ocorreu por meio de pequenos envelopes pardos, nos quais continha um papel em branco com um círculo (azul para clorexidina 0,12\% e laranja para chá de malva). Após o sorteio, o paciente era alocado em relação à primeira substância a ser utilizada. Os pacientes não tinham conhecimento sobre qual das substâncias estavam recebendo, apenas de que se tratava de um enxaguatório e de um chá. A intervenção ocorreu em todos os ciclos de quimioterapia. Os pesquisadores levavam, diariamente, durante o período de infusão da quimioterapia, o frasco contendo $30 \mathrm{ml}$ da substância para a realização dos bochechos juntamente de um pequeno copo de $10 \mathrm{ml}$, o qual deveria ser cheio a cada realização de bochecho, totalizando assim os $30 \mathrm{ml}$ por dia.

Todos os pacientes foram orientados sobre os procedimentos: a) bochecho três vezes ao dia (manhã, tarde e noite) durante o período de infusão da quimioterapia com 10 ml da substância determinada, durante 1 minuto, sendo posteriormente desprezada; b) preenchimento do diário durante dez dias após o término de cada ciclo de quimioterapia.

O diário, preenchido em nível domiciliar, continha perguntas fechadas sobre o nível de dor, de acordo com a Escala Visual Analógica (EVA) ${ }^{11}$, e sobre a autopercepção de sua mucosa oral, avaliado por meio de fotografias coloridas que exemplificavam os graus de mucosite oral. Assim, o paciente examinava a sua mucosa e verificava com qual foto ela se parecia mais. A cada ciclo, um novo diário foi entregue ao paciente.

Para caracterização da amostra, utilizaram-se estatísticas descritivas e porcentagem para analisar e comparar as variáveis entre os grupos em termos de frequência. Para comparaçáo de variáveis de desfecho selecionadas entre os grupos, utilizou-se o teste $t$ de Student. Todas as análises foram realizadas utilizando o programa estatístico Bioestat 5.0, adotando um nível de significância de $\mathrm{p} \leq 0,05$.

\section{RESULTADOS}

Fizeram parte da amostra estudada sete indivíduos, sendo $85,7 \%$ homens, com média de idade de $62,1 \pm 13,1$ [48-85] anos, caucasianos (85,8\%), 50\% ex-etilistas, $57,1 \%$ náo fumantes e $42,8 \%$ ex-fumantes. Destes, $28,5 \%$ realizaram a prática por mais de 15 anos. Em relação ao tipo histológico, $57,1 \%$ dos indivíduos apresentavam adenocarcinoma e $42,8 \%$ carcinoma de células escamosas, sendo o sítio topográfico mais prevalente o estômago.

Os sete sujeitos foram submetidos a um total de 18 ciclos de quimioterapia, os quais foram acompanhados pelos pesquisadores, sendo que todos os pacientes em todos os ciclos foram submetidos à infusão com 5-FU 
associado à cisplatina. Em dez ciclos, utilizou-se a clorexidina profilaticamente e, em oito, o chá de malva, de acordo com a randomização.

Ao analisar a idade, tempo de tabagismo e nível de dor entre os grupos, náo foram observadas diferenças significativas (Tabela 1).

A autopercepção dos pacientes em relação ao grau de sua mucosite oral, quando utilizaram clorexidina $0,12 \%$ como bochecho, variou entre grau 1 e 2 (Tabela 2). Quanto ao nível de dor referido por estes, a média mais alta encontrada foi de 2,6 no $7^{\circ}$ dia após a quimioterapia, seguido por 2,2 nos $6^{\circ}$ e $8^{\circ}$ dias (Tabela 3 ).

A autopercepção dos pacientes submetidos ao bochecho com chá de malva em relaçáo ao grau de sua mucosite oral variou entre grau 1 e 2 na maior parte dos dias, apresentando grau $3 \mathrm{em} 50 \%$ dos pacientes no $8^{\circ}$ dia após a infusão (Tabela 2). Quanto ao nível de dor referido por esses pacientes, a média mais alta encontrada foi de 2,9 no $6^{\circ}$ dia e 2,8 no $5^{\circ}$ dia pós-quimioterapia (Tabela 3 ).

\section{DISCUSSÃO}

Inúmeros estudos estão sendo realizados com o intuito de verificar os métodos mais eficazes para prevenção e tratamento da mucosite oral. Ainda não há um protocolo específico para prevenção dessa complicação e várias das substâncias estudadas são utilizadas empiricamente e de modo paliativo. Os estudos com essa finalidade são importantes, pois poderão determinar um protocolo específico e eficaz na prevenção da mucosite oral ${ }^{12,13}$.

Um dos métodos citados para prevenção e tratamento da mucosite oral é o digluconato de clorexidina $0,12 \%$, empregado em forma de bochechos ${ }^{5,14}$, profilaxia esta também utilizada neste estudo em função de suas propriedades antifúngica, antimicrobiana e anti-inflamatória ${ }^{6,7,8}$.

Além da clorexidina $0,12 \%$, também foi utilizado neste estudo o chá de malva. Apesar de a literatura não apresentar estudos relatando a utilização de chá de malva

Tabela 1. Comparação entre idade, tempo de tabagismo, grau de dor e grau de mucosite oral nos dias estudados entre os grupos clorexidina $(n=10)$ e chá de malva $(n=8)$

\begin{tabular}{|c|c|c|c|c|}
\hline Variável & Grupo & Média & Desvio-padrão & $\mathbf{p}$ \\
\hline \multirow{2}{*}{ Idade } & Clorexidina & 63,4 & 13,0 & 0,26 \\
\hline & Chá de malva & 65,8 & 15,8 & \\
\hline \multirow{2}{*}{ Tempo tabagismo } & Clorexidina & 2,7 & 2 & 0,26 \\
\hline & Chá de malva & 2,1 & 1,8 & \\
\hline \multirow{2}{*}{ Dor $1^{\circ}$ dia } & Clorexidina & 1 & 2 & 1,00 \\
\hline & Chá de malva & 1 & 1,2 & \\
\hline \multirow{2}{*}{ Dor $2^{\circ}$ dia } & Clorexidina & 1,6 & 2,5 & 0,86 \\
\hline & Chá de malva & 1,4 & 1,6 & \\
\hline \multirow{2}{*}{ Dor $3^{\circ}$ dia } & Clorexidina & 1,6 & 2,5 & 0,71 \\
\hline & Chá de malva & 2 & 2 & \\
\hline \multirow{2}{*}{ Dor $4^{\circ}$ dia } & Clorexidina & 2 & 3,1 & 0,86 \\
\hline & Chá de malva & 2,3 & 2,8 & \\
\hline \multirow{2}{*}{ Dor $5^{\circ}$ dia } & Clorexidina & 1,9 & 2,8 & 0,58 \\
\hline & Chá de malva & 2,8 & 3,4 & \\
\hline \multirow{2}{*}{ Dor $6^{\circ}$ dia } & Clorexidina & 2,2 & 2,5 & 0,67 \\
\hline & Chá de malva & 2,9 & 3,5 & \\
\hline \multirow{2}{*}{ Dor $7^{\circ}$ dia } & Clorexidina & 1,9 & 3,4 & 0,70 \\
\hline & Chá de malva & 2,4 & 3,3 & \\
\hline \multirow{2}{*}{ Dor $8^{\circ}$ dia } & Clorexidina & 2,2 & 3,3 & 0,97 \\
\hline & Chá de malva & 2,3 & 2,3 & \\
\hline \multirow{2}{*}{ Dor $9^{\circ}$ dia } & Clorexidina & 1,8 & 2,2 & 0,94 \\
\hline & Chá de malva & 1,9 & 2,3 & \\
\hline \multirow{2}{*}{ Dor $10^{\circ}$ dia } & Clorexidina & 1,9 & 2,5 & 0,93 \\
\hline & Chá de malva & 2 & 2,4 & \\
\hline \multirow{2}{*}{ Média grau de dor } & Clorexidina & 1,6 & 2,2 & 0,64 \\
\hline & Chá de malva & 2,1 & 2,3 & \\
\hline \multirow{2}{*}{ Média grau de mucosite oral } & Clorexidina & 1,6 & 0,3 & 0,76 \\
\hline & Chá de malva & 1,5 & 0,7 & \\
\hline
\end{tabular}


Tabela 2. Frequência (\%) dos graus de mucosite oral de acordo com a autopercepção dos pacientes nos dias estudados

\begin{tabular}{l|c|c|c|c|c|c|c|c}
\hline & \multicolumn{4}{|c|}{ Chá de malva } & \multicolumn{4}{c}{ Clorexidina 0,12\% } \\
\cline { 2 - 9 } & Grau 1 & Grau 2 & Grau 3 & Grau 4 & Grau 1 & Grau 2 & Grau 3 & Grau 4 \\
\hline $1^{\circ}$ dia & 100 & 0 & 0 & 0 & 88,9 & 11,1 & 0 & 0 \\
\hline $2^{\circ}$ dia & 100 & 0 & 0 & 0 & 55,6 & 33,3 & 11,1 & 0 \\
\hline $3^{\circ}$ dia & 100 & 0 & 0 & 0 & 55,6 & 44,4 & 0 & 0 \\
\hline $4^{\circ}$ dia & 50,0 & 50,0 & 0 & 0 & 25,0 & 62,5 & 12,5 & 0 \\
\hline $5^{\circ}$ dia & 50,0 & 50,0 & 0 & 0 & 37,5 & 37,5 & 12,5 & 12,5 \\
\hline $6^{\circ}$ dia & 50,0 & 50,0 & 0 & 0 & 33,3 & 33,3 & 22,2 & 11,1 \\
\hline $7^{\circ}$ dia & 50,0 & 50,0 & 0 & 0 & 37,5 & 50,0 & 0 & 12,5 \\
\hline $8^{\circ}$ dia & 50,0 & 0 & 50,0 & 0 & 50,0 & 37,5 & 12,5 & 0 \\
\hline $9^{\circ}$ dia & 66,7 & 0 & 33,3 & 0 & 62,5 & 37,5 & 0 & 0 \\
\hline $10^{\circ}$ dia & 66,7 & 33,3 & 0 & 0 & 62,5 & 25,0 & 12,5 & 0 \\
\hline
\end{tabular}

Tabela 3. Grau de dor nos 10 dias estudados nos ciclos que utilizaram chá de malva e clorexidina 0,12\%

\begin{tabular}{l|c|c|c|c}
\hline \multirow{2}{*}{} & \multicolumn{2}{|c|}{ Chá de malva } & \multicolumn{2}{c}{ Clorexidina 0,12\% } \\
\cline { 2 - 5 } & Média & $\begin{array}{c}\text { Desvio- } \\
\text {-padrão }\end{array}$ & Média & $\begin{array}{c}\text { Desvio- } \\
\text {-padrão }\end{array}$ \\
\hline $1^{\circ}$ dia & 1 & 1,2 & 1 & 2 \\
\hline $2^{\circ}$ dia & 1,4 & 1,6 & 1,6 & 2,5 \\
\hline $3^{\circ}$ dia & 2 & 2 & 1,6 & 2,5 \\
\hline $4^{\circ}$ dia & 2,3 & 2,8 & 2 & 3,1 \\
\hline $5^{\circ}$ dia & 2,8 & 3,4 & 1,9 & 2,8 \\
\hline $6^{\circ}$ dia & 2,9 & 3,5 & 2,2 & 2,5 \\
\hline $7^{\circ}$ dia & 2,4 & 3,3 & 2,6 & 3,4 \\
\hline $8^{\circ}$ dia & 2,3 & 3 & 2,2 & 3,3 \\
\hline $9^{\circ}$ dia & 1,9 & 2,3 & 1,8 & 2,2 \\
\hline $10^{\circ}$ dia & 2 & 2,4 & 1,9 & 2,5 \\
\hline
\end{tabular}

na prevenção da mucosite oral, esta é considerada uma erva medicinal e alimento funcional, com propriedades anti-inflamatória e antisséptica, em especial na cavidade oral, considerando seu uso externo em forma de bochecho ${ }^{8,9}$. Somando as propriedades citadas anteriormente, estudos in vitro mostraram que a Malva silvestrys $\mathrm{L}$ tem potencial antimicrobiano e antiaderente sobre micro-organismos da cavidade oral e antifúngica sobre quatro espécies de cepas de Candida $^{15}$, além de ser facilmente encontrada e ter um custo inferior comparada aos outros métodos de bochecho utilizados. Tais questóes, tornaram o chá de malva motivo de investigação neste estudo, além de ter propriedades semelhantes às da clorexidina $0,12 \%$.

Ainda neste estudo, tanto nos ciclos em que foi utilizada clorexidina quanto nos ciclos em que foi utilizado o chá de malva, houve uma menor severidade das lesôes, sugerindo a importância dessas substâncias nos estudos que visam ao estabelecimento de protocolos para prevenção de mucosite oral.
Mallick et al. ${ }^{5}$, por meio de um estudo duplo-cego, avaliaram 70 pacientes que realizaram bochechos durante o tratamento quimioterápico. Os resultados encontrados apontaram que aqueles que realizaram bochechos diários com digluconato de clorexidina $0,12 \%$, apesar de ter ocorrido a lesão, reduziram significativamente a incidência e a gravidade de mucosite, comparando com o grupo que não utilizou a solução para bochecho. Além disso, esses pacientes também apresentaram mais tempo para começar a apresentar a sintomatologia na mucosa oral. Entretanto, os autores não referem qual o quimioterápico utilizado. Um estudo semelhante foi realizado com 17 crianças de 2 a 12 anos em tratamento quimioterápico, as quais realizaram bochechos com digluconato de clorexidina $0,12 \%$, duas vezes ao dia, e desenvolveram mucosite oral de baixa gravidade ${ }^{16}$. Esses dados corroboram os achados neste estudo.

Apesar de a clorexidina mostrar resultados positivos em inúmeros estudos ${ }^{5,14,17,18}$, esta apresenta algumas características como o ardor e o sabor adstringente ${ }^{14} \mathrm{e}$ efeitos adversos que podem limitar seu uso, tais como: alteraçáo no paladar, escurecimento dental, feridas, irritaçôes na cavidade oral ${ }^{19}$ e sensação de queimação ${ }^{20}$. Porém, contrariamente a isso, em nosso estudo, não houve nenhum relato desses efeitos adversos pelos pacientes que fizeram o uso dessa substância profilaticamente.

Ao observar a autopercepção dos pacientes em relação aos seus graus de mucosite, verificou-se que, naqueles que utilizaram o chá de malva como profilaxia, náo houve relato de mucosite oral grau $4 \mathrm{em}$ nenhum dos dez dias de acompanhamento. Por outro lado, nos ciclos em que foi usada a clorexidina $0,12 \%$, houve um pequeno percentual de pacientes que relatou grau 4 nos $5^{\circ}, 6^{\circ}$ e $7^{\circ}$ dias.

Com relação ao sintoma de dor relatado pelos pacientes durante os dez dias após a quimioterapia, apesar de não ter sido significativo, a clorexidina pareceu apresentar 
menos sintomatologia dolorosa nos ciclos em que foi utilizada. Mas, em ambas as substâncias, a média máxima de dor apresentada foi verificada entre os $6^{\circ}$ e $7^{\circ}$ dias (grau 2,8 com o chá de malva e 2,6 com a clorexidina $0,12 \%)$, o que condiz com os resultados de Elyasi et al. ${ }^{14}$, que avaliaram um grupo experimental que fez o uso de clorexidina $0,2 \%$ e um grupo controle, evidenciando que o grupo experimental apresentou menos dor. Porém, a concentração da substância utilizada foi maior que em nosso estudo, e outro dado conflitante é o fato de que, além da clorexidina, os pacientes utilizaram também bochechos com soluçáo de cloreto de sódio e um protocolo de higiene oral rigoroso, cumprido por $90 \%$ dos pacientes.

No que diz respeito ao tempo da fase aguda da mucosite oral, as informaçóes são conflitantes, há autores que referem que ela ocorre entre os $7^{\circ}$ e $10^{\circ}$ dias após a quimioterapia ${ }^{13}$, enquanto outros relatam entre os $3^{\circ} \mathrm{e}$ $5^{\circ}$ dias após a quimioterapia ${ }^{4}$. Neste estudo, verificou-se que os dias de pico, os quais condizem com o nível de dor apresentado pelos pacientes, ocorreu entre os $5^{\circ}$ e $7^{\circ}$ dias, em ambas as substâncias profiláticas utilizadas.

Após instalada, o tratamento da mucosite oral é sintomático, dependendo do grau em que se apresenta, bem como da dor do paciente, a qual é subjetiva e varia de pessoa para pessoa. Quando necessário, recomenda-se o uso de analgésicos e anestésicos tópicos e analgésicos opioides, visando ao alívio da dor ${ }^{3,21}$. Neste estudo, nenhum dos pacientes relatou utilizar essas substâncias para alívio da dor durante os dez dias de acompanhamento mediante o diário.

A dor afeta a sobrevida do paciente, pois pode influenciar as capacidades orofaríngeas como alimentar-se normalmente, engolir, beber e falar ${ }^{21}$. Em nosso estudo, observou-se que, muito embora a dor e mucosite oral estiveram presentes, os pacientes não referiram, nas consultas de acompanhamento antes de cada ciclo de quimioterapia, grandes dificuldades para realizar essas funçôes orais, sugerindo, assim, a efetividade da clorexidina $0,12 \%$ e do chá de malva na redução dos sintomas da mucosite oral.

Entendemos que a forma de autoavaliação realizada neste estudo apresenta limitaçóes, uma vez que foi examinador dependente; ou seja, dependendo totalmente da percepção do próprio paciente. A fim de evitá-la, se deve sempre ressaltar ao paciente a realização do método com calma e cautela.

\section{CONCLUSÃO}

Tanto nos ciclos em que foi utilizada a clorexidina $0,12 \%$ quanto naqueles em que foi utilizado o chá de malva como profilaxia, a maioria dos pacientes apresentou a autopercepçáo do desenvolvimento de mucosite oral apenas nos graus 1 e 2 . Porém, nos ciclos em que a clorexidina foi utilizada, houve uma pequena ocorrência de mucosite oral grau 4. Não houve diferença estatística na sintomatologia dolorosa entre as duas substâncias.

\section{CONTRIBUIÇÕES}

Francielli Valduga e Elenusa Oltramari contribuíram na concepçáo do estudo, coleta de dados e redaçáo final. Letícia Tainá de Oliveira Lemes trabalhou na redação do estudo. Carlos Eduardo de Mattos trabalhou na coleta dos dados. Letícia Stefenon e Carolina Barreto Mozzini contribuíram na concepção do estudo, na metodologia e redação final.

\section{DECLARAÇÃO DE CONFLITO DE INTERESSES}

Nada a declarar.

\section{REFERÊNCIAS}

1. Baydar M, Dikilitas M, Sevinc A, Aydogdu I. Prevention of Oral Mucositis Due to 5-fluoracil Treatment with Oral Cryotherap. Journal of the National Medical Association 2005; 97(8):1161-1164.

2. Brito CA, Araujo DS, Granja JG, Souza SM, Lima MAG, Oliveira MC. Efeito da clorexidina e do laser de baixa potência na prevenção e no tratamento da mucosite oral. Rev Odontol UNESP 2012; 41(4):236-241.

3. Pourdeghatkar F, Montaghi M, Darbandi B, BagherSalami A. The Effect of Chamomile Mouthwash on the Prevention of Oral Mucositis Caused by Chemotherapy in Children with Acute Lymphoblastic Leukemia. Iranian Journal of Pediatric Hematology \& Oncology. 2017;7(2):76-81.

4. Sonis ST. Mucositis: the impact, biology and therapeutic opportunities of oral mucositis. Review. Oral Oncol. 2009; 45(12):1015-1020.

5 Mallick S, Benson R, Rath GK. Radiation induced oral mucositis: a review of current literature on prevention and management. European Archives of Oto-RhinoLaryngology: Official Journal of The European Federation of Oto-Rhino-Laryngological Societies (EUFOS): Affiliated with the German Society for OtoRhino-Laryngology. Head and Neck Surgery. 2016; 273(9):2285-2293.

6. Rastogi M, Khurana R, Revannasiddaiah S, Jaiswal I, Nanda SS, Gupta P, et al. Role of benzydamine hydrochloride in the prevention of oral mucositis in head and neck cancer patients treated with radiotherapy (>50Gy) with or without chemotherapy. Support Care Cancer. 2017; 25(5):1439-1443. 
7. Maekawa LE, Bringhenti FL, Lamping R, Oliveira LD, Marcacci S, Koga-Ito CY. Atividade antimicrobiana de enxaguatórios bucais sem álcool a base de clorexidina sobre Candida albicans. Rev Odontol UNESP 2010; 39(1):15-19.

8. Brasil. Agência Nacional de Vigilância Sanitária. Formulário de fitoterápicos da Farmacopéia Brasileira. Brasília, DF: Anvisa; 2011.

9. Gasparetto JC, Martins CAF, Hayashi SS, Otuky MF, Pontarolo R. Ethnobotanical and scientific aspects of Malva sylvestris L.: a millennial herbal medicine. J Pharm Pharmacol.2011; 64:172-189.

10. Carneiro Neto JN, Menezes JDS, Moura LB, Massucato EMS, Andrade CR. Protocols for management of oral complications of chemotherapy and/or radiotherapy for oral cancer: Systematic review and meta-analysis current. Med Oral Patol Oral Cir Bucal 2017; 22(1):e15-23.

11. Price DD, McGrath PA, Raffi A, Buckingham B. The validation of visual analogue scales as ratio scale measures for chronic and experimental pain. Pain .1983; 17:45-56.

12. Hosseinjani H, Hadjibabaie M, Gholami K, Javadi M, Radfar M, Jahangard-Rafsanjani Z, et al. The efficacy of erythropoietin mouthwash in prevention of oral mucositis in patients undergoing autologous hematopoietic SCT: a double-blind, randomized, placebo-controlled trial. Hematol Oncol. 2017; 35(1):106-112.

13. Volpato LER, Silva TC, Oliveira TM, Sakai VT, Machado MAAM. Mucosite bucal rádio e quimioinduzida. Rev Bras Otorrinolaringol 2007; 73(4):562-568.

14. Elyasi S, Hosseini S, Niazi-Moghadam MR, Aledavood SA, Karimi G. Effect of Oral Silymarin Administration on Prevention of Radiotherapy Induced Mucositis: A Randomized, Double-Blinded, Placebo-Controlled Clinical Trial. Phytother Res. 2016; 30(11):1879-1885.

15. Alves PM, Queiroz LMG, Pereira JV, Pereira MSV. Atividade antimicrobiana, antiaderente e antifúngica in vitro de plantas medicinais brasileiras sobre microrganismos do biofilme dental e cepas do gênero Candida. Rev. Soc. Bras. Med. Trop. 2009; 42(2):222-224.

16. Soares A, Aquino ARL, Carvalho CHP, Nonaka CFW, Almeida D, Pereira-Pinto L. Frequency of Oral Mucositis and Microbioloical Analysis in Children with Acute Lymphoblastic Leukemia Treated with 0,12\% Chlorhexidine Gluconate. Braz Dent J. 2011; 22(4):312-316.

17. Antunes HS, Ferreira EMS, Faria LMD, Schirmer M, Rodrigues PC, Small IA, et al. Streptococcal bacteremia in atients submitted to hematopoietic stem cell transplantation: The role of tooth brushing and use of chlorhexidine. Med Oral Patol Oral Cir Bucal. 2010; 15(2):303-309.

18. Lanzos I, Herrera D, Santos S, O’ Connor A, Penã C, Lanzos C, et al. Mucositis in irradiated cancer patients: Effects of an antiseptic mouthrinse. Med Oral Patol Oral Cir Bucal. 2010; 15(5):732-738.
19. Mccoy L, Wehler CJ, Rich SE, Garcia RI., Miller DR, Jones JA. Adverse events associated with chlorhexidine use. JADA. 2008; 139:178-183.

20. Hayashi H, Kobayashi R, Suzuki A, Yamada Y, Ishida M, Shakui T, et al. Preparation and clinical evaluation of a novel lozenge containing polaprezinc, a zinc-Lcarnosine, for prevention of oral mucositis in patients with hematological cancer who received high-dose chemotherapy. Med Oncol. 2016; 33(8):91-98.

21. Ala S, Saeedi M, Janbabai G, Ganji R, Azhdari E, Shiva A. Efficacy of Sucralfate Mouth Wash in Prevention of 5-fluorouracil Induced Oral Mucositis: A Prospective, Randomized, Double-Blind, Controlled Trial. Nutr Cancer. 2016;68(3):456-463. 